\title{
Delayed bleeding after prophylactic bilateral internal iliac artery balloon occlusion in elective caesarean hysterectomy for the management of placenta accreta
}

\author{
Abdul Karim Othman ${ }^{1 *}$, Noraslawati Razak ${ }^{2}$, Mohd Hanif Che Mat ${ }^{2}$
}

\begin{abstract}
${ }^{1}$ Faculty of Medicine, University Sultan Zainal Abidin, Kuala Terengganu, Terengganu, Malaysia
${ }^{2}$ Department of Anaesthesia and Intensive Care, Hospital Sultanah Nurzahirah, Kuala Terengganu, Terengganu, Malaysia
\end{abstract}

Received: 21 January 2017

Revised: 28 January 2017

Accepted: 28 February 2017

\section{*Correspondence:}

Dr. Abdul Karim Othman,

E-mail: neooptima@yahoo.com.my

Copyright: (c) the author(s), publisher and licensee Medip Academy. This is an open-access article distributed under the terms of the Creative Commons Attribution Non-Commercial License, which permits unrestricted non-commercial use, distribution, and reproduction in any medium, provided the original work is properly cited.

\begin{abstract}
Morbidly adherent placenta (MAP) can be divided into placenta accrete, placenta increta and placenta percreta. It is associated with high parity, multifetal gestation, advanced maternal age, assisted reproductive technologies, placenta previa, and more importantly a history of caesarean section or uterine surgery. Globally, the incidence of placenta accrete has increased and seems to be in parallel with the increasing rate of caesarean section delivery.

Despite rapidly evolving diagnostic imaging, and growing of surgical expertise, morbidly adherent placenta (MAP) remains an important cause of maternal morbidity and mortality, especially related with life-threatening postpartum haemorrhage. Although the choice of treatment for placenta accrete is puerperal hysterectomy, this procedure itself involves a greater risk of intra-operative haemorrhage.

Elective caesarean hysterectomy using prophylactic bilateral internal iliac artery balloon occlusion offer an interesting approach which can minimize the risk of intra-operative haemorrhage. However, our case report describes the case of a 28-year old Gravida 3 Para 2 morbidly obese parturient diagnosed to have placenta previa type 3 posterior with accrete who experienced a complication of life threatening massive bleeding post-operatively after an elective caesarean hysterectomy using a prophylactic bilateral internal iliac artery balloon occlusion intra-operatively.
\end{abstract}

Keywords: Morbidly adherent placenta, Maternal morbidity, Mortality, Placenta accrete

\section{INTRODUCTION}

Morbidly adherent placenta (MAP) is a disorder of placenta implantation that develops when the loss of decidua basalis allowing an uncontrolled invasion of the chrorionic villi into the myometrium. ${ }^{1}$ Based on the depth of invasion, MAP can be divided into three categories: placenta accreta, placenta increta, and placenta percreta. The least severe form of MAP, placenta accrete involves superficial placenta invasion, while the most severe form of MAP, placenta percreta involves placenta invasion through the uterine serosa with possible extension into the adjacent organs. ${ }^{2}$

Risk factors associated with MAP include high parity, multifetal gestation, advanced maternal age, assisted reproductive technologies, placenta previa, and most importantly a history of caesarean section or other uterine surgery. ${ }^{1}$ Globally, the incidence of placenta accrete has increased and seems to parallel the increasing caesarean delivery rate. The incidence has increased sharply from 1 in 533 pregnancies for the periods of 1982-2002 to 1 in 4 
027 pregnancies in the 1970s, and to 1 in 2510 pregnancies in the $1980 \mathrm{~s}^{3-5}$ In a 2015 review of birth registries, Mehrabadi et al, noted an incidence of placenta accrete of 14.4 per 10000 deliveries. ${ }^{6}$ Myometrial damage caused by a previous caesarean delivery with either anterior or posterior placenta previa overlying the uterine scar contribute the greatest risk for developing placenta accrete. Silver et al, reported that in the presence of placenta previa, the risk of placenta accrete was $3 \%$, $11 \%, 40 \%, 61 \%$, and $67 \%$ for the first, second, third, fourth and fifth or greater repeat caesarean deliveries, respectively. $^{7}$

Despite rapidly evolving diagnostic imaging and surgical technologies, and growing of surgical expertise, MAP remains an important cause of maternal morbidity and mortality, especially related with life-threatening postpartum haemorrhage with a $7 \%$ risk of maternal mortality. ${ }^{1}$ Although the choice treatment for placenta accrete is puerperal hysterectomy, it involves greater risk of intraoperative haemorrhage. ${ }^{8}$ The bleeding is usually treated with bilateral ligation of the uterine arteries or the internal iliac arteries. ${ }^{9}$ However, due to the vast network of collateral arteries in the pelvis, particularly during pregnancy, adequate control of this bleeding is possible in less than $42 \%$ of the cases, particularly when there is involvement of the adjacent structures, such as bladder, colon, and ureter. All these will lead to massive intraoperative bleeding and significantly increased the morbidity and mortality rates. ${ }^{10}$

We describe here the case of a 28-year old G3P2 morbidly obese parturient diagnosed to have placenta previa Type 111 posterior with accreta who experienced a complication of delayed massive bleeding after an elective caesarean hysterectomy using a prophylactic bilateral internal iliac artery balloon occlusion intraoperatively.

\section{CASE REPORT}

A case of 28 -year-old obese (BMI $42 \mathrm{~kg} / \mathrm{m} 2)$ gravida 3 para 2 pregnant women at 37 weeks' period of amenorrhoea, who was diagnosed with a Placenta Previa Type 111 with accreta. This is her third pregnancy in which both of her previous deliveries was ended with lower segment caesarean section (LSCS). Both caesarean sections were done under subarachnoid block with an uneventful intra-operatively. She had a medical background of Essential Hypertension and bronchial asthma which are controlled with pharmacological treatment. She was planned by her obstetrician for elective caesarean hysterectomy with an internal iliac artery balloon catheterization, inserted by an interventional radiologist.

Her pre-anaesthetic review was done by the anaesthetic team on day 5 and day 1 prior to her admission to the antenatal ward. The operation was planned supported with Intensive Care Unit (ICU) care post-operatively, the high-risk consent and a team from blood bank were informed regarding the risk of massive bleeding from the surgical site which may necessitate the activation of Massive Transfusion Protocol (MTP). Video-assisted laryngoscope (C-mac) and troop pillow were made available in Operation Theatre (OT) as part of her potential difficult airway management.

On the morning prior to elective caesarean section, the patient was sent to Invasive Cardiac Lab for the insertion of bilateral internal iliac artery occlusion balloon. After completing the insertion, the balloon was deflated and the patient was sent to operating theatre for elective caesarean hysterectomy. The procedure was done uneventfully.

She was induced for general anaesthesia using rapid sequence induction technique with cricoid pressure using Sellick manoeuvre. Her induction and intubation were uneventful and large bore venous access was inserted after the induction. A midline skin incision with a fundal midline hysterotomy incision was done. Intra-operatively, mean arterial pressure (MAP) was maintained above $70 \mathrm{mmHg}$. After the delivery of the baby, occlusion balloon catheter was inflated and proceed with elective hysterectomy. Hemodynamically remains stable with an estimated blood loss was approximately $800 \mathrm{mls}$. After completion of hysterectomy, the occlusion balloon was deflated. There were no active bleedings seen at the surgical site and the catheter was remove at the end of the surgery. The total duration of inflation balloon was 60 minutes starting from the delivery of the baby to the completion of hysterectomy.

In the immediate post-operative period, she was extubated to facemask oxygen. Hemodynamic parameters remain stable and no blood or blood products transfusion was required. She was discharged from recovery ward to a high dependency suite for the continuation of postoperative observation and management. The patient was discharged well from high dependency suite to general ward 8 hours postoperatively. In general ward patient's condition was stable and noted to be ambulating well. However, 15 hours after elective caesarean hysterectomy, patient start complaining abdominal pain and shoulder pain. She was examined by the obstetric team and found to be in hypovolemic shock. She was pale, tachycardia, hypotensive and her abdomen was distended.

Re-laparotomy was issued immediately with an intraoperative finding of massive blood and blood clot collection with a bleeding originated from left uterine artery and from the hysterectomy stump. Reinforcement suturing and bilateral internal iliac artery ligation was done. Estimated blood loss was 9 liters and patient was transfused 7 pints' whole blood together with 1 cycle of DIVC regime (6-unit cryoprecipitate, 4 unit of platelets and 4 units of fresh frozen plasma). Post-operatively, the patient was sent to ICU for post-operative observation 
and management and subsequently was extubated to ventimask oxygen 6 hours post re-laparotomy. The patient was discharged well to general ward after 24 hours' observation in ICU without any complication or organ impairment.

\section{DISCUSSION}

In reporting this complication, we do not wish to discredit the potential benefit of prophylactic bilateral internal iliac artery balloon occlusion in the management of placenta accrete. Rather we wish to raise the awareness regarding the possible and potential complications associated with its use, the importance of establishing clear safety protocols to reduce its complications and to regulate a standard protocol in monitoring patient during the immediate post-operative period.

Paull et al first reported the use of infrarenal aortic balloon occlusion to minimize massive obstetric haemorrhage in $1995 .{ }^{11}$ This is followed by prophylactic internal iliac artery balloon occlusion which was successfully used in two patients with placenta percreta. ${ }^{8}$ The goal of internal iliac artery balloon occlusion is to temporarily occlude the internal iliac arteries in order to reduce uterine perfusion and decrease the rate of blood flow, thereby reducing blood loss, improving visualization during surgery, allowing for urgent arterial embolization (if require), and possibly preventing hysterectomy. ${ }^{12}$ However, until now there is still a lack of significant evidence showing the benefit in terms of haemorrhage control and uterus preservation. Current literature has been limited to case reports (Sewell et al; Greenberg et al; Bishop et al; Matsueda et al), descriptive case series (Teixidor Vinas et al, Carnevale et al; Cho et al). Small cases control comparisons (Shivastava et al), or small prospective cohort studies (Bodner et al), which have yielded mixed results. ${ }^{13}$

Failed occlusion of the internal iliac arteries which is often due to extensive anastomosis in the pelvic vasculature and technique-related complications have been contributed to the controversy over this procedure. Vascular complications, such as popliteal artery thrombosis and iliac artery thrombosis occurred in 3 of $19(15.8 \%)$ (2 of $13(15.4 \%), 1$ of 27 subjects $(3.7 \%)$ (Teixida Vinas) and 2 of 21 subjects $(9.5 \%) .{ }^{14}$ The hypercoagulable state of pregnancy increased the risk of a thromboembolic event, and the vascular wall remodelling of arteries in pregnant women also enhances the complication and susceptibility of dissection. Another vascular complication is ruptured of an internal iliac artery caused by endovascular balloons (Jessica P.S. et al). ${ }^{15,16}$ Although this complication is a technique-related complication, it's significantly compromising patient safety by causing a massive haemorrhage perioperatively. Perioperative massive haemorrhage also occurred after removing the pre-set balloon immediately after caesarean section resulting in a significant blood loss and requiring emergent embolization and hysterectomy based on the fact that $66 \%$ of haemorrhage occur within 2 hours after delivery and $33 \%$ of haemorrhage occur more than 4 hours after delivery. ${ }^{17}$ Certain issues that need to be highlighted which arise from our case report which include:

- Timing for deflation of the occlusion balloon and removal of the catheter. Prolonged catheterisation is a significant contributing factor to vascular complications while early device removal may lead to uncontrolled massive haemorrhage and inability to occlude the internal iliac arteries for controlling the haemorrhage as the device was already removed. Until now, there is no definitive guideline for determining the duration of inflation and the appropriate time for device removal.

- The timing for allowing ambulation after surgery. Keeping the lower extremities strictly still is the priority advice to patients during the period of device placement, however, for some patients it may be difficult or uncomfortable for them to follow the recommendation. Failure to follow this recommendation will lead to vascular complication as agitation and twisting of the lower extremities can contribute to the displacement of the catheter and damaging the blood vessels. As for our case report, the patient was ambulating in the general ward within few hours after the elective hysterectomy. Patient ambulating with the possibility of surgicaltechnique failure may trigger the slow bleeding from the surgical site leading to a massive haemorrhage. Again, until now there is no definitive time interval for the patients to start ambulating after the surgery.

- The requirement to challenge the surgical site for the potential of bleeding by increasing the blood pressure before removing the device and closing the abdomen. The controlled of bleeding at the surgical site may be masked by a lower blood pressure induced by general anaesthesia. Failure to perform this simple challenge may lead to potential haemorrhage during the postoperative period when patient's blood pressure is normalized or elevated by any reason.

- Postoperative monitoring for early detection of massive haemorrhage and thromboembolic event to the lower extremities is not standardised. Both of these complications can lead to a major morbidity and mortality to the patients especially in late detection Thus a standard post-operative care and monitoring is urgently need. A standard postoperative care and monitoring should be developed in order to prevent any delayed in detection in both of these potential complications. A set of monitoring which include a certain hours of strict bed rest, tools to monitor any potential thromboembolic event to the lower extremities such as a serial examination and monitoring of skin perfusion and sensation, and also a tools to monitor for potential massive haemorrhage such as a more frequent hemodynamic monitoring and serial arterial 
blood gases analysis for the first few hours after the surgery in order to observe a trend of haemoglobin level to the detect the presence of worsening metabolic acidosis as an early indicator for the possibility of ongoing or worsening occult haemorrhage.

The safety and effectiveness of preoperative occlusive balloon catheters for abnormal placenta remains controversial. We strongly encouraged this novel technique based on our previous experiences and report from various case-reports and studies, but we strongly advise for caution and safety exercised during the whole perioperative period given the lack of meaningful evidence to support the strategy, and the occurrence of potential major practice-related morbidity and mortality vascular complications and post procedure massive haemorrhage. Further research is needed to support the standardization of the proposed method in clinical application, especially in the monitoring of the patients during the immediate postoperative period.

\section{ACKNOWLEDGEMENTS}

Author would like to thank all multidisciplinary members of the obstetrics, interventional radiology, anaesthetic teams, and intensive care unit teams for their dedications and support.

Funding: No funding sources Conflict of interest: None declared

Ethical approval: Not required

\section{REFERENCES}

1. Committee on Obstetric Practice. Committee Opinion No. 529: Placenta accrete. Obstet Gynecol. 2012;120:207.

2. Badner LJ, Nosher JL, Gribbin C, Siegel RL, Beala S, Scotza W. Balloon-assisted occlusion of the internal iliac arteries in patients with placenta accrete/percreta. Cardiovase Intervent Radiol. 2006;29:354-61

3. Wu S, Kocherginsky M, Hibbard JU. Abnormal placentation: twenty-year analysis. Am J Obstet Gynecol. 2005;192:1458-61.

4. Read JA, Cotton DB, Miller FC. Placenta accrete: changing clinical aspects and outcome. Obstet Gynecol. 1980;56:31-4.

5. Miller DA, Chollet JA, Goodwin TM. Clinical risk factors for placenta previa-placenta accreta. Am J Obstet Gynecol. 1997;177(1):210-4.

6. Mehrabadi A, Hutcheon JA, Liu S, Bartholomew S, Kramer MS, Liston RM. Contribution of placenta accrete to the incidence of post-partum haemorrhage. Obstet Gynecol. 2015;125:814-21.

7. Silver RM, Landon MB, Rouse Dj, Leveno KJ, Seong CY, Thom EA, et al. Maternal morbidity associated with multiple repeat caesarean deliveries. Obstet Gynecol. 2006;107:1226-32.

8. Dubois J, Garel L, Grignon A, Lemay M, Leduc L. Placenta-percreta: balloon occlusion and embolization of the internal iliac arteries to reduce intraoperative blood losses. Am J Obstet Gynecol. 1997;176:723-6.

9. Evans S, McShane P. The efficacy of internal iliac artery ligation in obstetric haemorrhage. Surg Gynecol Obstet. 1985;160:250-3.

10. Clark SL, Phelan JP, Yeh SY, Bruce SR, Paul RH. Hypogastric artery ligation for obstetric haemorrhage. Obstet Gynecol. 1985;66:353-6.

11. Paull JD, Smith J, Williams L, Davison G, Devine T, Holt M. Balloon occlusion of the abdominal aorta during caesarean hysterectomy for placenta percreta. Anaesth Intensive Care.1995;23(6):731-4.

12. Dilauro MD, Dason S, Wagner Y. Prophylactic balloon occlusion of internal iliac arteries in women with placenta accreta: literature review and analysis. Clin. Rad. 2012;67:515-20.

13. Shrivavastava V, Nageotte M, Major C, Haydon M, Wing D. Case-control comparison of caesarean hysterectomy with and without prophylactic placement of intravascular balloon catheters for placenta accreta. Am J Obstet Gynecol. 2007;197(4):401-5.

14. Chou MM, Kung HF, Hwang JI, Chen WC, Tseng JJ. Temporary prophylactic intravascular balloon occlusion of the common iliac arteries before caesarean hysterectomy for controlling operative blood loss in abnormal placentation. Taiwan J. Obstet Gynecol. 2015;54(5):493-8.

15. Jessica PS, Sukhbir SS, Cleve Z. Internal iliac artery rupture caused by endovascular balloons in a woman with placenta percreta. J. Obstet Gynecol Can. 2016;38(11):1024-7.

16. Gagnon J, Boucher L, Kaufman I, Brown R, Moure A. Iliac artery rupture related to balloon insertion for placenta accreta causing maternal haemorrhage and neonatal compromise. Can J. Anaesth. 2013;60:1212-7.

17. Teixidor VM, Chandraharan E, Moneta MV, Belli AM. The role of interventional radiology in reducing haemorrhage and hysterectomy following caesarean section for morbidly adherent placenta. Clin Radiol. 2014;69(8):345-51.

Cite this article as: Othman AK, Razak N, Mat MHC. Delayed bleeding after prophylactic bilateral internal iliac artery balloon occlusion in elective caesarean hysterectomy for the management of placenta accreta. Int J Reprod Contracept Obstet Gynecol 2017;6:1656-9. 